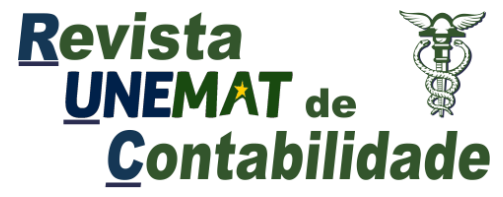

v. 9, n. 17,2020

\title{
AGRESSIVIDADE TRIBUTÁRIA E A INFORMATIVIDADE DOS LUCROS
}

\section{TAX AGGRESSIVINESS AND EARNINGS INFORMATIVENESS}

\author{
Nome Antonio Lopo Martinez \\ Instituição/Afiliação Universidade de Coimbra \\ País Portugal \\ Contato principal para correspondência. \\ Nome Eduardo Augusto Viana Duarte \\ Instituição/Afiliação Fucape Business School \\ País Brasil
}

\begin{abstract}
RESUMO
O presente estudo investiga a associação entre a agressividade tributária e a informatividade dos lucros, está compreendida como a capacidade do lucro influenciar no preço da ação no mercado brasileiro. Metodologicamente, foram feitos testes utilizando um modelo de regressão preço-lucro, que mensura a informatividade dos lucros através do ERC (coeficiente de resposta dos lucros) de 2005 a 2016 com um total de 1879 observações. Resultados indicam que empresas mais agressivas tributariamente, medidas pelo ETR (taxa efetiva de tributação), apresentam ERC negativos ou mais baixos e empresas menos agressivas apresentaram ERC positivos ou mais altos. Essa pesquisa tem como contribuição, evidenciar um tipo de custo não tributário associado com a agressividade fiscal, materializado em menor informatividade dos lucros, sendo esta uma dimensão da qualidade dos lucros. Esses achados são relevantes para usuários externos, deixando explicito que quando uma empresa adota uma postura que desvia da padrão normal de agressividade tributária, isso afeta diretamente o grau de informatividade dos lucros no Brasil.
\end{abstract}

Palavras-chave: Agressividade tributária; informatividade dos lucros; taxa de tributação efetiva; coeficiente de resposta dos lucros.

\begin{abstract}
The present study addresses the relationship between tax avoidance and earnings informativeness, where informativeness is understood as the capacity of earnings to influence the price of the share in the Brazilian market. Methodologically, tests were made using a priceprofit regression model, which measures the informativity of profits through the ERC (earnings response coefficient) from 2005 to 2016, a total of 1879 observations. Results indicate that companies that are more tax aggressive, measured by the ETR (effective tax rate), present negative or lower ERC and less tax aggressive companies had positive or higher ERC. This research has as its contribution, to provide evidence of a type of non-tax cost associated with tax aggressiveness, materialized in a lower informativity of profits, a important dimension of earnings quality. These findings are relevant to external users, leaving explicit that when a company adopts a posture that off takes the normal pattern of tax aggressiveness, this directly affects the degree of earnings informativeness in Brazil.
\end{abstract}

Keywords: Tax Aggressiveness; earnings informativeness; effective tax rate, earnings response coefficient. 


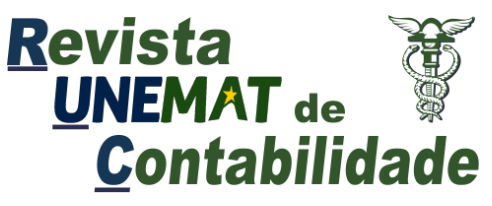

v. 9, n. 17,2020

\section{INTRODUÇÃO}

O estudo sobre a relevância das informações contábeis está cada vez mais sendo objeto de pesquisas nacionais. Na literatura internacional encontra-se uma vasta quantidade de pesquisas sobre a relevância das informações contábeis.

Esse estudo verifica a associação entre a agressividade tributária e a informatividade dos lucros. A temática agressividade tributária tem sido objeto de diversos estudos no Brasil, apreciando causas e consequências desse comportamento (Martinez, 2017). Por sua vez a informatividade dos lucros avalia a sensibilidade do preço de ações ao lucro, sendo um importante métrica para avaliação da qualidade do lucro (Dechow, Ge, \& Schrand, 2010).

O objetivo da pesquisa é analisar a relação da agressividade fiscal com a informatividade dos lucros no mercado acionário brasileiro. Este estudo considera as questões de transparência relacionadas às atividades de agressividade fiscal como as principais causadoras da perda da informatividade dos lucros. Balakrishnan, Blouin e Guay (2012) argumentam que a agressividade fiscal reduz significativamente a transparência de uma empresa ao aumentar a complexidade financeira e organizacional.

A motivação principal dessa pesquisa é saber se de alguma maneira o fato de a empresa ser mais ou menos agressiva impacta em como o lucro se reflete nos preços. Este estudo vai informar se no Brasil as empresas que são mais agressivas acabam comprometendo a informatividade dos lucros. Essa pesquisa informa ao algum usuário externo, se ao adotar uma postura mais ou menos agressiva, como que isso vai refletir na informatividade dos lucros, na qualidade, e na capacidade de refletir os preços.

A pesquisa é composta por duas etapas. Na primeira investiga-se se as empresas com níveis elevados de agressividade fiscal (planejamento tributário refletido por taxa efetivas de tributação baixa), apresentam uma associação negativa com a informatividade dos lucros (medida pelo ERC). Em uma segunda etapa, inclui-se o efeito da adoção do IFRS (International Accounting Standards Board) no Brasil, se antes e/ou após a adoção, teve influência nos resultados.

Metodologicamente, foi utilizada como proxy de agressividade fiscal a taxa efetiva de tributação (ETR - effective tax rate). Considera-se que o ETR reflete melhor qualquer atividade destinada a reduzir a carga fiscal de uma empresa (Hanlon \& Heitzman, 2010).

Os resultados indicam que empresas mais agressivas estão associadas negativamente com a informatividade dos lucros, medida pelo ERC. Empresas que se mantem perto da faixa estatuária do Brasil de 34\%, são mais informativas. Teste feito também com o efeito da adoção do IFRS, apresenta resultados parecidos obtidos sem a utilização desse efeito.

Este estudo contribui para a literatura brasileira sobre a qualidade dos lucros, fornecendo novas evidências de que existe um custo não tributário associado ao planejamento tributário, isto é materializado na perda da informatividade dos lucros. As informações transmitidas aos investidores através dos números contábeis desempenham um papel fundamental no processo de precificação de ações e, portanto, à determinação do custo de capital de uma empresa. A pesquisa tem implicações para empresas envolvidas no planejamento tributário eficiente, que incorpora custos explícitos e não tributários (Scholes et al., 2009).

A perda da informatividade dos lucros é uma métrica sobre a qualidade destes, e até agora não se tinha relacionado com a agressividade fiscal. No entanto, tem-se verificado um crescente interesse em estudos sobre custos não tributários e questões de transparência relacionadas com tributos (Balakrishnan, Blouin, \& Guay, 2012). 


\section{Revista \\ UNEMAT de \\ Contabilidade}

v. 9 , n. 17,2020

\section{REFERENCIAL TEÓRICO}

Hanlon e Heitzman (2010) definem a agressividade tributária com uma redução da carga fiscal através de conjunto de atos e ações, estrategicamente planejados visando a redução do ônus tributário . Por sua vez, Chen, Chen, Cheng e Shevlin (2010) chegam a definição idêntica acerca da agressividade tributária. Nessas ações para se fazer um planejamento podem se usar medidas licitas ou medidas abusivas, consideradas ilegais. A agressividade tributária não se trata de ilegalidade, e sim de mecanismos de dentro da legislação para se utilizar do gerenciamento de resultados e diminuir o lucro tributável (Lisowsky, Leslie, \& Andrew, 2013). Lietz (2013), em sua pesquisa, afirma que as empresas se utilizam de variedades de técnicas para reduzir a sua carga tributária, sendo difícil classificarem-se essas ações por possuírem diversas proxies.

Para ser considerado um planejamento tributário eficiente, deve-se ter em conta três questões: all parts, all taxes, all costs, ou seja, todas as partes envolvidas, todos os tributos e todos os custos (Scholes et al. 2009). Existe uma variedade de custos relacionados a um plano tributário, podendo-se dividi-los em custos explícitos e custos não tributários. Os primeiros incluem despesas para o desenvolvimento, implementação e monitoramento de estratégias de um plano fiscal tais como custos trabalhistas diretos ou indiretos, custos de auditoria, custos do sistema de informação, custos de coordenação entre unidades de negócios, bem como taxas ou penalidades que possam surgir na medida em que as agressividades fiscais são consideradas inapropriadas perante as autoridades responsáveis (Hoopes, Mescall, \& Pittman, 2012).

Os gestores enfrentam regularmente uma trade-off entre decisões financeiras e fiscais, uma vez que o seu desejo de reportar baixos lucros tributáveis para as autoridades fiscais entra em conflito com o objetivo de reportat aos acionistas altos lucros financeiros, cria-se assim um custo não tributário, materializado na percepção negativa do mercado, quando ser reporta baixo lucros para atender fins tributários (Scholes, Wilson, \& Wolfson, 1990). Os custos de agência, política e reputação também são igualmente considerados custos não tributários (Gallemore, Maydew, \& Thornock, 2012).

Pode-se encontrar na literatura estudos que questionam em que medidas o mercado valoriza os métodos da agressividade fiscal. Desai e Dharmapala (2009) fornecem provas de grande amostra em que a agressividade fiscal é geralmente uma função da governança corporativa e que os investidores das respectivas empresas atribuem valor positivo para as medidas de agressividade fiscal.

Balakrishnan, Blouin e Guay (2012) investigaram possíveis questões de transparência financeira que podem estar associadas ao planejamento tributário agressivo. Os resultados sugerem que a agressividade fiscal pode aumentar a opacidade (ausência de transparência) do ambiente global de informação financeira das empresas. Alguns estudos são motivados pela perspectiva da agência sobre a agressividade fiscal e propõem que esta é usada por gestores oportunistas para mascarar os resultados (Desai \& Dharmapala, 2009).

Conforme Desai, Hogam e Wilkins (2006), as complexas transações tributárias usadas para diminuir os gastos com impostos levam os gestores a prejudicar não apenas os acionistas diretamente, gerenciando parte dos lucros da empresa, mas também indiretamente, criando estruturas que reduzem a informatividade deles.

A informatividade dos lucros é uma métrica muito utilizada na qualidade destes, por descrever a capacidade dos ganhos para auxiliar os acionistas no processo de precificação das ações de uma empresa, fornecendo informações úteis para uma tomada de decisão (Dechow, Ge, \& Schrand, 2010). 


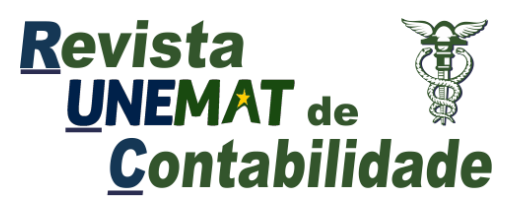

v. 9, n. 17,2020

De acordo com a teoria contábil, as informações podem ser consideradas uteis se tiverem implicações de avaliação, ou seja, se isso for fazer com que os investidores ajustem os preços das ações (Zimmerman, 1983). O resultado relatado de uma empresa segundo Ball e Brown (1968), tem um conteúdo informacional considerável, o que explica o uso frequente do coeficiente de resposta do lucro (ERC - Earnings Response Coefficient) como uma proxy para a qualidade dos lucros. O ERC mede a reação dos preços das ações, uma resposta prejudicada dos lucros ou uma resposta mais fraca do que a esperada pode ser interpretada como uma perda da informatividade dos lucros (Collins \& Kothari,1989).

As empresas utilizam uma variedade de estratégias de planejamento tributário com o objetivo de reduzir o gasto com tributos; consequentemente, tais reduções de tributos levarão a um aumento de fluxo de caixa. Em primeira análise, pode-se dizer que isso tem um efeito positivo no valor de uma empresa (Desai \& Dharmapala, 2009). No entanto, o planejamento tributário também gera custos, sejam eles explícitos, sejam implícitos (Scholes, Wolfson, Erickson, Maydew, \& Shevlin, 2009).

Kubata, Lietz e Watrin (2013) pesquisaram o mercado americano e encontraram evidências de que quanto maior a agressividade fiscal das empresas menor é a informatividade dos lucros, um tipo de custo não tributário.

Existem argumentos válidos no sentido de que a agressividade fiscal reduz a transparência financeira e organizacional das empresas, o que pode prejudicar a informatividade dos lucros (Balakrishnan, Blouin, \& Guay, 2012).

De acordo com a teoria apresentada, a pesquisa possui a seguinte hipótese:

H1: A agressividade fiscal está negativamente associada a informatividade dos lucros

A H1 estabelece uma relação entre a agressividade fiscal e a informatividade dos lucros, para levantar a hipótese de que os lucros serão prejudicados em sua informatividade à medida que os níveis de agressividade aumentem.

Para apurar a medida em que os lucros fornecem informações úteis aos acionistas, as análises baseiam-se em reações de preços (respostas), associadas às mudanças dos lucros. $\mathrm{O}$ coeficiente de resposta do lucro (ERC - Earnings Response Coefficient) tem sido usado frequentemente nas pesquisas para medir a informatividade dos lucros. A informação é útil para os acionistas se tiver implicações de avaliação, ou seja, se essas informações os levarem a ajustar os seus preços (Zimmerman, 1983).

Com base nessa premissa, o coeficiente de resposta do lucro (ERC - Earnings Response Coefficient) estima o conteúdo informativo dos lucros na medida em que uma mudança nos lucros afeta o preço das ações (Collins \& Kothari, 1989). De acordo com H1, as empresas que apresentam uma taxa efetiva de tributação (ETR - effective tax rate) baixa tendem a demonstrar coeficiente de resposta (ERC - Earnings Response Coefficient) menor, indicando que a agressividade fiscal está associada negativamente com a informatividade dos lucros.

Com base na pesquisa seminal de Ball e Brown (1968), frequentemente se investigam as respostas do mercado de ações aos lucros para compreender a qualidade destes. Seguindo essa prática no âmbito do mercado de capitais, a pesquisa empírica utiliza o seguinte modelo de preço-lucro:

$$
\text { preço }_{i}=\beta_{0}+\beta_{1} L P A_{i}+\varepsilon_{i}
$$

Em que Preço é o preço da ação e $L P A$ reflete o lucro por ação. Levando em consideração a teoria de precificação e em linha com a hipótese do mercado eficiente, os preços 


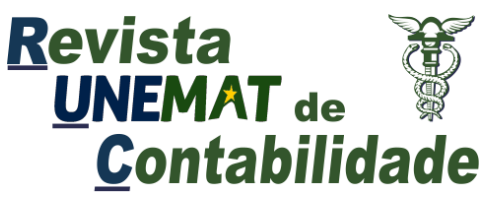

v. 9, n. 17,2020

das ações podem ser assumidos de forma agregada para refletir todas as informações de valor relevante. Segue-se que o coeficiente de inclinação $\beta 1$ é o coeficiente de resposta dos lucros (ERC - Earnings Response Coefficient). A teoria implica que a resposta dos lucros dos investidores é um indicador direto da informatividade dos lucros (Holthausen \& Verrecchia, 1988).

\section{METODOLOGIA}

A pesquisa é dotada de característica descritiva; o método a ser utilizado é o quantitativo, por meio de técnicas estatísticas. A metodologia para testar a hipótese apresentada foi adaptada do trabalho de Kubata, Lietz e Watrin (2013).

A amostra selecionada foram companhias de capital aberto listadas na B3. Os dados de lucro por ação e preço por ação foram coletados do Economática por um período de 10 anos, de 2005 a 2014. Foram utilizadas 384 empresas, excluindo as empresas financeiras, somando um total de 1879 observações.

Ainda através da mesma base de dados, acessaram-se, nas demonstrações dos resultados, as informações do lucro antes do imposto de renda (LAIR) e as despesas de imposto de renda (IRPJ) e contribuição social sobre o lucro líquido (CSLL), para medir a taxa efetiva de tributação (ETR - effective tax rate).

A taxa efetiva de tributação (ETR - effective tax rate) é uma das medidas utilizadas para se determinar o nível de agressividade fiscal (Dunbar, Higgins, Phillips, \& Plesko, 2010). A taxa de tributação efetiva (ETR - effective tax rate) é calculada através da divisão da soma da despesa imposto de renda e a contribuição social pelo lucro líquido antes dos impostos (LAIR) (Martinez \& Dalfior, 2015).

Para observar separadamente as empresas que se envolvem em níveis diferentes de agressividade fiscal, todas as amostras são agrupadas de acordo com o seu nível de taxa efetiva de tributação (ETR - effective tax rate). São divididos em 12 níveis diferentes, de acordo com a seguinte sequência:

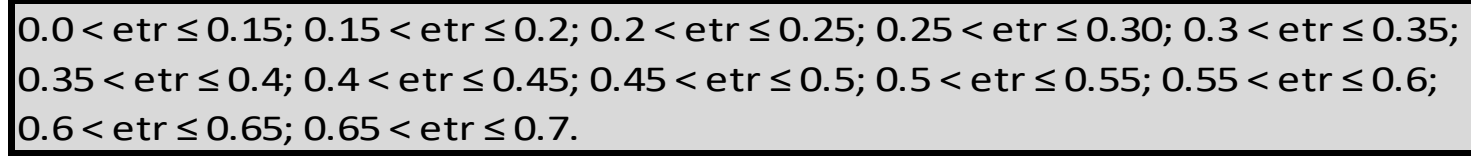

Os modelos são estimados para cada um dos 12 níveis de agressividade fiscal. As empresas que apresentaram taxa efetiva de tributação (ETR - effective tax rate) acima de 70\% foram excluídas, por não permitirem uma boa análise econômica; já aquelas que apresentaram taxa efetiva de tributação (ETR - effective tax rate) menor que $0 \%$ também foram desconsideradas, para controle de outliers e viés da amostra.

É importante ressaltar que a agressividade fiscal diminui à medida que a métrica cresce, ou seja, níveis mais altos de ETR implicam menores níveis de agressividade fiscal.

O objetivo de separar em níveis é para mostrar se diferenças de ETR mais ou menos agressivas têm influência no ERC. Separados, pode-se analisar, para cada grupo de nível de ETR, se o coeficiente aumenta ou diminui de acordo com a taxa de tributação efetiva.

Foi utilizada a Equação (2) a seguir para medir o coeficiente de resposta do lucro (ERC - Earnings Response Coefficient) no Brasil: 


$$
\operatorname{preço~}_{i}=\beta_{0}+\beta_{1} L P A_{i}+\varepsilon_{i}
$$

Preço é o preço da ação;

$L P A$ reflete o lucro por ação;

$\beta_{1}$ é o coeficiente de resposta dos lucros (ERC - Earnings Response

\section{Coefficient).}

Outro teste foi investigar se após a adoção da IFRS (International Accounting Standards Board), teve impacto significativo nos resultados. A adoção do padrão internacional de normas contábeis (IFRS) tem como objetivo melhorar a transparência e a comparabilidade das informações financeiras entre as empresas segundo Hope, Yiqiang e Tony (2005), tornando seu mercado financeiro mais acessível aos investidores estrangeiros.

Barth (2010) realizou uma investigação com empresas de 23 países, na qualverificou que as empresas que adotaram as IFRS apresentaram maior qualidade na informação contábil.

Ainda com o propósito de responder à questão de pesquisa foi estimado a Equação 3 como segue:

$$
\text { preço }_{i}=\beta_{0}+\beta_{1} L P A_{i}+\beta_{2} \text { DummyIFRS }_{i}+\beta_{3} L P A * \text { DummyIFRS }_{i}+\varepsilon_{i}
$$

Preço é o preço da ação;

$L P A$ reflete o lucro por ação;

DummyIFRS assume 0 para antes da IFRS e 1 para após IFRS

$L P A * D u m m y$ IFRS lucro por ação após a IFRS

O que torna consoante no modelo acima são os coeficientes, $\boldsymbol{\beta}_{\mathbf{1}}$ e $\boldsymbol{\beta}_{\mathbf{3}}$ com as seguintes interpretações:

$E R C_{2005-2007}=\boldsymbol{\beta}_{\mathbf{1}}-$ mede o impacto nos preços antes da adoção da IFRS.

$E R C_{2008-2014}=\boldsymbol{\beta}_{\mathbf{1}}+\boldsymbol{\beta}_{\mathbf{3}}-$ mede o impacto nos preços após a adoção da IFRS.

\section{RESULTADOS}

\subsection{ESTATISTICA DESCRITIVA}

A Tabela 1 apresenta a estatística descritiva do ETR, LPA e Preço, segmentado por nível de ETR. Nota-se que a média do ETR nos níveis é compatível às faixas descritas na figura 1,0 que torna relevante nas análises as estatísticas associadas ao LPA e Preço por nível.

\begin{tabular}{|c|c|c|c|c|c|c|c|}
\hline \multirow[b]{2}{*}{ Nível } & \multirow[b]{2}{*}{ Variável } & \multirow[b]{2}{*}{ Obs. } & \multicolumn{5}{|c|}{ Estatísticas } \\
\hline & & & Média & $\begin{array}{l}\text { Desvio } \\
\text { padrão }\end{array}$ & Mediana & Mínimo & Máximo \\
\hline \multirow{3}{*}{ Nível 1} & $\overline{\text { ETR }}$ & \multirow{3}{*}{421} & 0,078 & 0,045 & 0,079 & 0,000 & 0,150 \\
\hline & LPA & & $-12,640$ & 87,785 & 0,794 & $-1202,903$ & 436,833 \\
\hline & Preço & & 23,238 & 43,436 & 10,415 & 0,020 & 437,444 \\
\hline \multirow{3}{*}{ Nível 2} & ETR & \multirow{3}{*}{205} & 0,175 & 0,014 & 0,176 & 0,150 & 0,200 \\
\hline & LPA & & 1,401 & 10,119 & 1,083 & $-110,049$ & 39,519 \\
\hline & Preço & & 31,184 & 69,679 & 12,290 & 0,014 & 500,846 \\
\hline \multirow{3}{*}{ Nível 3} & ETR & \multirow{3}{*}{268} & 0,226 & 0,015 & 0,228 & 0,200 & 0,250 \\
\hline & LPA & & 1,045 & 8,293 & 0,947 & $-87,008$ & 38,268 \\
\hline & Preço & & 22,404 & 52,438 & 10,535 & 0,125 & 618,994 \\
\hline Nível 4 & ETR & 332 & 0,277 & 0,015 & 0,278 & 0,251 & 0,300 \\
\hline
\end{tabular}

Tabela 1 - Estatística descritivas dos indicadores por Nível de ETR 


\begin{tabular}{|c|c|c|c|c|c|c|c|}
\hline & LPA & & 1,294 & 6,898 & 1,311 & $-79,002$ & 33,851 \\
\hline & Preço & & 28,804 & 67,716 & 14,675 & 0,187 & 682,407 \\
\hline & ETR & & 0,326 & 0,015 & 0,328 & 0,300 & 0,349 \\
\hline Nível 5 & LPA & 335 & 3,496 & 23,226 & 1,210 & $-53,859$ & 382,243 \\
\hline & Preço & & 28,858 & 61,824 & 13,457 & 0,203 & 552,982 \\
\hline & ETR & & 0,371 & 0,014 & 0,370 & 0,350 & 0,400 \\
\hline Nível 6 & LPA & 149 & 0,526 & 13,876 & 0,687 & $-145,443$ & 36,488 \\
\hline & Preço & & 29,748 & 78,712 & 11,984 & 0,228 & 526,010 \\
\hline & ETR & & 0,422 & 0,015 & 0,421 & 0,401 & 0,449 \\
\hline Nível 7 & LPA & 62 & $-0,026$ & 7,705 & 0,501 & $-39,003$ & 18,813 \\
\hline & PRECO & & 24,933 & 52,572 & 10,593 & 0,261 & 299,600 \\
\hline & ETR & & 0,474 & 0,014 & 0,473 & 0,450 & 0,496 \\
\hline Nível 8 & LPA & 38 & 0,096 & 2,513 & 0,452 & $-11,660$ & 3,619 \\
\hline & PRECO & & 15,590 & 15,914 & 9,788 & 1,163 & 82,000 \\
\hline & ETR & & 0,527 & 0,015 & 0,529 & 0,502 & 0,549 \\
\hline Nível 9 & LPA & 26 & 1,150 & 2,138 & 0,231 & $-0,700$ & 7,928 \\
\hline & PRECO & & 27,073 & 39,712 & 12,533 & 1,199 & 188,382 \\
\hline & ETR & & 0,574 & 0,017 & 0,570 & 0,551 & 0,599 \\
\hline Nível 10 & LPA & 18 & $-0,456$ & 2,755 & 0,131 & $-9,996$ & 2,540 \\
\hline & PRECO & & 42,166 & 96,951 & 18,837 & 0,812 & 424,703 \\
\hline & ETR & & 0,618 & 0,013 & 0,615 & 0,600 & 0,643 \\
\hline Nível 11 & LPA & 15 & 0,379 & 1,465 & 0,092 & $-1,247$ & 5,329 \\
\hline & PRECO & & 18,532 & 17,406 & 12,823 & 0,301 & 60,756 \\
\hline & ETR & & 0,667 & 0,013 & 0,663 & 0,651 & 0,690 \\
\hline Nível 12 & LPA & 10 & $-1,946$ & 7,618 & 0,095 & $-23,154$ & 4,584 \\
\hline & PRECO & & 26,891 & 33,758 & 20,077 & 4,276 & 120,000 \\
\hline & ETR & & 0,250 & 0,128 & 0,257 & 0,000 & 0,690 \\
\hline Total Geral & LPA & 1879 & $-1,631$ & 43,616 & 0,897 & $-1202,903$ & 436,833 \\
\hline & PRECO & & 26,605 & 59,501 & 12,017 & 0,014 & 682,407 \\
\hline
\end{tabular}

Fonte: Elaborado pelos autores.

Nota : Onde Nível 1: 0,00<ETR $\leq 0,15$, Nível 2: $0,15<\mathrm{ETR} \leq 0,20 ;$ Nível 3: $0,20<\mathrm{ETR} \leq 0,25$; Nível 4: $0,25<\mathrm{ETR} \leq 0,30 ; \quad$ Nível 5: $0,30<\mathrm{ETR} \leq 0,35 ;$ Nível 6: 0,35<ETR $\leq 0,40$; Nível 7: $0,40<\mathrm{ETR} \leq 0$,45; Nível 8: $0,45<$ ETR $\leq 0,50$; Nível 9: 0,50<ETR $\leq 0,55$; Nível 10: 0,55<ETR $\leq 0,60$; Nível 11: $0,60<\mathrm{ETR} \leq 0,65$ : Nível 12 : $0,65<\mathrm{ETR} \leq 0,70$.

Dos doze níveis expostos, quatro deles - níveis 1, 7, 10 e 12 - incidiram com a média de lucro por ação negativa, tendo como destaque o nível 1, com a maior média e o maior desvio padrão. Também no nível 1,50\% das observações incidiram com LPA abaixo de 0,794. Ao avaliar a média de preço nesses níveis, destaca-se o nível 10, com a maior média de preço e, igualmente, a maior variabilidade (desvio padrão).

Analisando os demais níveis - LPA médio positivo, apresentam-se com as maiores médias de LPA e preço os níveis 2 e 5, tendo este último exibido maior variabilidade, ou seja, observações não homogêneas.

No geral, a média do ETR incidiu em 0,25, com desvio padrão em torno da média de 0,128. A média do LPA sucedeu em -1,631 e a mediana de 0,897. Por fim, o preço, a média incidiu em 26,60, com desvio padrão de 59,501.

\subsection{MODELO DE REGRESSÃO}

De acordo com a H1, o principal objetivo e testar a associação entre a agressividade fiscal e a informatividade dos lucros. A amostra é organizada de acordo com os diferentes níveis de ETR. Os intervalos variam de 0,0 a 0,7 . A tabela 2 apresenta os resultados da análise da associação entre a agressividade fiscal e a informatividade dos lucros. 
Tabela 2 - Preço por LPA para diferentes níveis de ETR - Equação (2)

\begin{tabular}{cccccccc}
\hline Nível & ETR & ERC & $\begin{array}{c}\text { Erro } \\
\text { Padrão }\end{array}$ & Estatística t & Valor p & R-quadrado & Observações \\
\hline Nível 1 & $0,00-0,15$ & $-0,081$ & 0,024 & $-3,390$ & $\mathbf{0 , 0 0 1} * * *$ & $2,7 \%$ & 421 \\
Nível 2 & $0,15-0,20$ & 0,187 & 0,483 & 0,390 & 0,699 & $0,1 \%$ & 205 \\
Nível 3 & $0,20-0,25$ & $-1,517$ & 0,376 & $-4,030$ & $\mathbf{0 , 0 0 0 * * *}$ & $5,8 \%$ & 268 \\
Nível 4 & $0,25-0,30$ & $-3,492$ & 0,505 & $-6,910$ & $\mathbf{0 , 0 0 0 * * *}$ & $12,7 \%$ & 332 \\
Nível 5 & $0,30-0,35$ & 0,863 & 0,138 & 6,250 & $\mathbf{0 , 0 0 0 * * *}$ & $10,5 \%$ & 335 \\
Nível 6 & $0,35-0,40$ & 0,855 & 0,463 & 1,850 & $\mathbf{0 , 0 6 6 *}$ & $2,3 \%$ & 149 \\
Nível 7 & $0,40-0,45$ & 2,104 & 0,838 & 2,510 & $\mathbf{0 , 0 1 5} * *$ & $9,5 \%$ & 62 \\
Nível 8 & $0,45-0,50$ & $-1,103$ & 1,039 & $-1,060$ & 0,296 & $3,0 \%$ & 38 \\
Nível 9 & $0,50-0,55$ & 1,471 & 2,315 & 6,360 & 0,524 & $7,5 \%$ & 26 \\
Nível10 & $0,55-0,60$ & 8,106 & 8,563 & 0,950 & 0,358 & $5,3 \%$ & 18 \\
Nível11 & $0,60-0,65$ & 1,626 & 3,264 & 0,500 & 0,627 & $1,9 \%$ & 15 \\
Nível12 & $0,65-0,70$ & $-4,106$ & 0,589 & $-6,970$ & $\mathbf{0 , 0 0 0 * * *}$ & $85,9 \%$ & 10 \\
\hline
\end{tabular}

Nota: $* * *, * * e *$ significativo ao nível de $1 \%, 5 \%$ e $10 \%$ respectivamente.

Modelo1: preço $_{i}=\beta_{0}+\beta_{1} L P A_{i}+\varepsilon_{i}$ e ERC $=\boldsymbol{\beta}_{1}$.

Fonte: Elaborado pelos autores.

Os resultados da Tabela 2 são obtidos a partir de regressões seguindo o modelo 1. Estatisticamente, é possível avaliar a relação entre o Preço e Lucro (LPA) por nível de ETR. Ao analisar as estatísticas, nota-se que o coeficiente de resposta ao lucro (ERC) resultou em uma estimativa negativa nas faixas de ETR que variam de 0,0 a 0,30 (níveis 1, 3, e 4), o que revela que empresas mais agressivas fiscalmente, apresentam lucros com pouca informatividade consistente com a $\mathrm{H} 1$.

Pode-se destacar que, os níveis 1, 3 e 4 são empresas dentro da faixa descrita por Martinez e Dalfior (2015) como "empresas mais agressivas" - ETR inferior a 34\%, ou seja, a agressividade fiscal está negativamente associada à informatividade dos lucros.

As empresas com ETR próximas da taxa de tributação estatutária do Brasil de $34 \%$ (níveis 5,6 e 7) apresentam ERC positivos indicando que empresas menos agressivas são mais informativas. A ETR dessas empresas varia de 0,30 a 0,45 .

Empresas que apresentam altos níveis ETR (nível 12) que variam 0,65 a 0,70, estão associadas de forma semelhante as empresas com a ETR de níveis baixos. Dhaliwal, Lee e Farguer (1991) mostram que a capacidade dos lucros de fornecer informações relevantes para o mercado é reduzida para empresas que estão alavancadas, assumindo que parte desses lucros são usados para pagar dívida em vez de serem repassados aos acionistas.

Para tentar explicar a informatividade dos lucros para as empresas com ETR altos (níveis 8 a 12) que variam entre 0,45 a 0,70 , estes intervalos de observações devem ser interpretados com cuidado, por possuírem um número bem pequeno da amostra e em certos níveis como o 10 e 11 que variam de 0,55 a 0,65 não apresentaram significância estatística.

Ainda com propósito de responder as indagações da presente pesquisa, foi estimado o modelo 2, cujos resultados estão expostos na Tabela 3. Não diferente do modelo 2, neste modelo permanece o foco de investigar a relação entre o Preço e Lucro (LPA) por nível de ETR, com o acréscimo da dummyIFRS, isto é, mitigar se a informatividade do lucro foi influenciada ou não após a adoção da IFRS. 
Tabela 3 - Preço por LPA para diferentes níveis de ETR com DummyIFRS - Equação (3)

\begin{tabular}{|c|c|c|c|c|c|c|c|c|}
\hline Nível & ETR & Variável & ERC & $\begin{array}{c}\text { Erro } \\
\text { Padrão } \\
\end{array}$ & $\begin{array}{c}\text { Estatística } \\
\mathrm{t}\end{array}$ & Valor $\mathrm{p}$ & $\begin{array}{c}\text { R- } \\
\text { quadrado }\end{array}$ & Observações \\
\hline \multirow{3}{*}{ Nível1 } & \multirow{3}{*}{$\begin{array}{c}0,00- \\
0,15\end{array}$} & LPA & $-0,126$ & 0,063 & $-2,010$ & $0,045 * *$ & \multirow{3}{*}{$2,8 \%$} & \multirow{3}{*}{421} \\
\hline & & DummyIFRS & $-0,961$ & 6,215 & $-0,150$ & 0,877 & & \\
\hline & & LPA*DummyIFRS & 0,053 & 0,068 & 0,780 & 0,434 & & \\
\hline \multirow{3}{*}{ Nível2 } & \multirow{3}{*}{$\begin{array}{c}0,15- \\
0,20\end{array}$} & LPA & 1,327 & 2,439 & 7,100 & $0,000 * * *$ & \multirow{3}{*}{$20,6 \%$} & \multirow{3}{*}{205} \\
\hline & & DummyIFRS & 37,566 & 13,345 & 2,820 & $0,005 * * *$ & & \\
\hline & & LPA*DummyIFRS & $-1,726$ & 2,478 & $-7,150$ & $\mathbf{0 , 0 0 0 * * *}$ & & \\
\hline \multirow{3}{*}{ Nível3 } & \multirow{3}{*}{$\begin{array}{c}0,20- \\
0,25\end{array}$} & LPA & 2,212 & 1,946 & 1,140 & 0,257 & \multirow{3}{*}{$7,6 \%$} & \multirow{3}{*}{268} \\
\hline & & DummyIFRS & 13,961 & 8,481 & 1,650 & 0,101 & & \\
\hline & & LPA*DummyIFRS & $-3,868$ & 1,983 & $-1,950$ & $0,052 *$ & & \\
\hline \multirow{3}{*}{ Nível4 } & \multirow{3}{*}{$\begin{array}{c}0,25- \\
0,30\end{array}$} & LPA & 3,540 & 3,279 & 1,080 & 0,281 & \multirow{3}{*}{$14,5 \%$} & \multirow{3}{*}{332} \\
\hline & & DummyIFRS & 28,995 & 11,618 & 2,500 & $\mathbf{0 , 0 1 3} * *$ & & \\
\hline & & LPA*DummyIFRS & $-7,17$ & 3,318 & $-2,160$ & $\mathbf{0 , 0 3 1} * *$ & & \\
\hline \multirow{3}{*}{ Nível5 } & \multirow{3}{*}{$\begin{array}{c}0,30- \\
0,35\end{array}$} & LPA & 4,708 & 0,687 & 6,850 & $0,000 * * *$ & \multirow{3}{*}{$18,5 \%$} & \multirow{3}{*}{335} \\
\hline & & DummyIFRS & 14,392 & 8,520 & 1,690 & $\mathbf{0 , 0 9 2 *}$ & & \\
\hline & & LPA*DummyIFRS & $-3,994$ & 0,700 & $-5,700$ & $\mathbf{0 , 0 0 0 * * *}$ & & \\
\hline \multirow{3}{*}{ Nível6 } & \multirow{3}{*}{$\begin{array}{c}0,35- \\
0,40\end{array}$} & LPA & 5,709 & 4,891 & 1,170 & 0,245 & \multirow{3}{*}{$4,2 \%$} & \multirow{3}{*}{149} \\
\hline & & DummyIFRS & 28,988 & 17,286 & 1,680 & 0,096 & & \\
\hline & & LPA*DummyIFRS & $-4,866$ & 4,913 & $-0,990$ & 0,324 & & \\
\hline \multirow{3}{*}{ Nível7 } & \multirow{3}{*}{$\begin{array}{c}0,40- \\
0,45\end{array}$} & LPA & 12,946 & 4,510 & 2,870 & $0,006 * * *$ & & \\
\hline & & DummyIFRS & 23,632 & 24,679 & 0,960 & 0,342 & $18,2 \%$ & 62 \\
\hline & & LPA*DummyIFRS & $-11,247$ & 4,586 & $-2,450$ & $0,017 * *$ & & \\
\hline & & LPA & 13,068 & 9,572 & 1,370 & 0,181 & & \\
\hline Nível8 & $\begin{array}{c}0,45- \\
050\end{array}$ & DummyIFRS & 17,377 & 9,552 & 1,820 & $\mathbf{0 , 0 7 8 *}$ & $12,1 \%$ & 38 \\
\hline & & LPA*DummyIFRS & $-14,237$ & 9,627 & $-1,480$ & 0,148 & & \\
\hline & & LPA & 5,455 & 2,190 & 2,490 & 0,210 & & \\
\hline Nível9 & $0,00-$ & DummyIFRS & $-0,164$ & 10,561 & $-0,020$ & 0,988 & $12,8 \%$ & 26 \\
\hline & & LPA*DummyIFRS & 17,342 & 2,755 & 6,290 & 0,152 & & \\
\hline & & LPA & 2,376 & 88,815 & 0,030 & 0,979 & & \\
\hline Nível10 & $\begin{array}{c}0,55- \\
060\end{array}$ & DummyIFRS & 31,674 & 137,928 & 0,230 & 0,822 & $7,8 \%$ & 18 \\
\hline & & LPA*DummyIFRS & 7,593 & 89,326 & 0,080 & 0,933 & & \\
\hline & & LPA & $-3,273$ & 12,345 & $-0,270$ & 0,796 & & \\
\hline Nível11 & $0,00-$ & DummyIFRS & $-10,388$ & 10,334 & $-1,010$ & 0,336 & $11,2 \%$ & 15 \\
\hline & & LPA*DummyIFRS & 5,937 & 12,851 & 0,460 & 0,653 & & \\
\hline & & LPA & $-54,120$ & 100,253 & $-0,540$ & 0,609 & & \\
\hline Nível12 & $\begin{array}{c}0,05- \\
070\end{array}$ & DummyIFRS & 11,661 & 13,701 & 0,850 & 0,427 & $87,4 \%$ & 10 \\
\hline & & LPA*DummyIFRS & 50,074 & 100,255 & 0,500 & 0,635 & & \\
\hline
\end{tabular}

Nota: $* * *, * * e *$ significativo ao nível de $1 \%, 5 \%$ e $10 \%$ respectivamente.

Modelo2: preço $_{i}=\beta_{0}+\beta_{1}$ LPA $_{i}+\beta_{2}$ DummyIFRS $_{i}+\beta_{3} L P A *$ DummyIFRS $_{i}+\varepsilon_{i} e$

$E R C_{2005-2007}=\boldsymbol{\beta}_{1}$ e $E R C_{2008-2014}=\boldsymbol{\beta}_{\mathbf{1}}+\boldsymbol{\beta}_{3}$

Fonte: Elaborado pelos autores

Analisando coeficiente de resposta dos lucros (ERC) do Modelo $2-E R C_{2005-2007}=\boldsymbol{\beta}_{1}$ e $E R C_{2008-2014}=\boldsymbol{\beta}_{\mathbf{1}}+\boldsymbol{\beta}_{\mathbf{3}}$, evidencia-se que a informatividade dos lucros foi impactada significativamente após IFRS.

Antes de 2008, ou seja, antes da adoção do IFRS no Brasil, as empresas que apresentam ETR de 0,0 a 0,15 (nível 1) apresentaram o ERC ( $\left.\boldsymbol{\beta}_{\mathbf{1}}\right)$ negativo de -0,126, após a adoção da IFRS o ERC $\left(\boldsymbol{\beta}_{\mathbf{1}}+\boldsymbol{\beta}_{\mathbf{3}}\right)$ não apresentou significância estatística. Empresas com ETR de 0,15 a

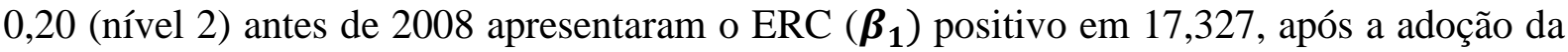
IFRS o ERC $\left(\boldsymbol{\beta}_{\mathbf{1}}+\boldsymbol{\beta}_{\mathbf{3}}\right)$ apresentou um coeficiente negativo de $-0,399$. Os nível 3 e 4 que são as empresas que apresentam ETR de 0,20 a 0,30 apresentaram o ERC $\left(\boldsymbol{\beta}_{\mathbf{1}}\right)$ sem significância estatística antes de 2008 , após a adoção da IFRS o ERC $\left(\boldsymbol{\beta}_{\mathbf{1}}+\boldsymbol{\beta}_{\mathbf{3}}\right)$ do nível $3(0,20$ a 0,25) 


\section{Revista \\ UNEMAT de \\ Contabilidade}

v. 9, n. 17,2020

apresentou um valor negativo de $-1,656$ e o nível $4(0,25$ a 0,30) também apresentou um valor negativo de -3,629. Resultados muitos parecidos com os do modelo 1, indicando que após a IFRS empresas que mantem seu ETR abaixo de 34\%, alíquota estatuária do Brasil, apresentam lucros com menos informatividade, sugerindo que a agressividade fiscal está associada negativamente com a informatividade dos lucros.

Empresas do nível 5 e 7 com ETR de 0,30 a 0,35 e 0,40 a 0,45 respectivamente, apresentam o ERC $\left(\boldsymbol{\beta}_{\mathbf{1}}\right)$ positivo de 4,708 para o nível 5 e 5,709 para o nível 7, após a adoção da IFRS o ERC $\left(\boldsymbol{\beta}_{\mathbf{1}}+\boldsymbol{\beta}_{\mathbf{3}}\right)$ continuou positivo 0,714 para o nível 5 e 1,699 para o nível 7 . Empresas com ETR de 0,35 a 0,40 (nível 6) não apresentaram significância estatísticas nos resultados. Novamente os resultados indicam que após a adoção da IFRS, empresas que se mantem próximas a alíquota estatuária do Brasil apresentam ERC positivos, indicando maior informatividade nos lucros.

Já empresas com níveis altos de ETR de 0,45 a 0,70 não apresentaram significância estatística em seus resultados. Por se tratarem de uma parte da amostra com baixa observações, ficaria de difícil interpretação desses resultados.

Os resultados no geral evidenciam que a informatividade do lucro é maior nos valores próximos das taxas de tributação estatutárias sobre lucro, ou seja de 34\% . Quanto mais próximo a taxa de tributação efetiva esteja daquela prescrita na legislação mais informativo será o lucro. Desvios em relação a essa taxa de $34 \%$ conduzem a uma menor informatividade do lucro. Essa relação é reforçada após a adoção do IFRS.

\section{CONCLUSÕES}

A redução na informatividade dos lucros pode representar um tipo de custo não tributário relacionados ao planejamento tributário das empresas. Os resultados apresentados indicam que níveis mais altos de agressividade tributária estão associados a uma perda na informatividade dos lucros medido pelo ERC (coeficiente de resposta do lucro). Na amostra empresas com níveis de ETR entre 0,0 a 0,30 (empresas consideradas agressivas) apresentam ERC negativos. Já empresas com ETR próximos a faixa estatutária (34\%), ou seja, empresas consideradas menos agressivas com níveis de ETR entre 0,30 a 0,45, apresentam ERC positivos.

Adicionalmente, foi estimado um modelo para investigar a relação entre o Preço e o Lucro por ação, acrescentando uma variável dummyIFRS, com o objetivo de analisar se houve impacto no ERC antes e após a adoção do IFRS. Resultados encontrados são muito semelhantes com o primeiro modelo estimado, entretanto que com a adoção do IFRS, os resultados são reforçados, ou seja, a perda de informatividade é acentuada nos desvios das taxas de tributação efetiva em relação a taxa estatutária. Empresas mais agressivas apresentaram ERC negativos indicando que, níveis altos de agressividade fiscal apresentam pouca informatividade em seus lucros e da mesma forma como no modelo 1 estimado, empresas com faixas de ETR próximas a alíquota estatuária do Brasil, apresentaram coeficientes de resposta (ERC) positivo, indicando que nesse grupo de empresas a informatividade dos lucros é maior.

A informação transmitida aos investidores através dos números contábeis desempenha um papel crucial no processo de precificação de ações e no custo de capital de uma empresa. Consequentemente, os resultados documentados nesta pesquisa têm implicações para as empresas envolvidas em estratégias agressivas planejamento tributário, que incorporam tributos implícitos e custos não tributários (Scholes et al. [2009]). Deve se ter muito cuidado em ponderar se as poupanças fiscais explícitas geradas pela elisão fiscal, justificam os seus efeitos 


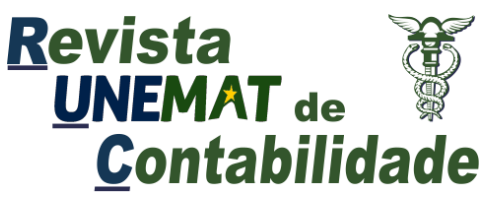

v. 9, n. 17,2020

negativos sobre a informatividade dos lucros. Pode ser que esses fatores conduzam algumas empresas para restringir sua agressividade tributária.

Pesquisas futuras que lidam com as consequências da agressividade fiscal e seus efeitos no mercado de capitais, poderiam acrescentar variáveis de controles não utilizadas nessa pesquisa, para identificar outros fatores que impactam no preço, em particular, poderia ainda tentar identificar mais precisamente o comprometimento da informatividade pela agressividade fiscal. Além disso, uma análise adicional seria segregar o lucro líquido entre o seu fluxo de caixa e as acumulações (accruals) para estudar os efeitos de cada componente. Isso pode gerar uma melhor compreensão das fontes subjacentes e potenciais direcionadores de qualidade do lucro. Em particular, perspectivas interessantes sobre quais ações específicas de planejamento tributário poderiam ser o principal condutor da perda de informatividade do lucros.

\section{REFERENCIAS}

Balakrishnan, K., Blouin, J. L., \& Guay, W. R. (2012). Does tax aggressiveness reduce corporate transparency?.Working paper, University of Pennsylvania, 2012.

Ball, R., \& Brown, P. (1968). An empirical evaluation of accounting income numbers. Journal of accounting research, 6(2), 59-178.

Barth, M. (2010). International accounting standards and accounting quality. Working Paper.

Chen, S., Chen, X., Cheng, Q., \& Shevlin, T. (2010). Are family firms more tax aggressive than non-family firms?. Journal of Financial Economics, 95(1), 41-61.

Collins, D. W., \& Kothari, S. P. (1989). An analysis of intertemporal and cross-sectional determinants of earnings response coefficients. Journal of accounting and economics, 11(1), 143-181.

Dechow, P., Ge, W., \& Schrand, C. (2010). Understanding earnings quality: A review of the proxies, their determinants and their consequences. Journal of accounting and economics, 50(2-3), 344-401.

Desai, H., Hogan, C. E., \& Wilkins, M. S. (2006). The reputational penalty for aggressive accounting: Earnings restatements and management turnover. The Accounting Review, 81(1), 83-112.

Desai, M. A., \& Dharmapala, D. (2009). Corporate tax avoidance and high-powered incentives. Journal of Financial Economics, 91(3), 537-546.

Dhaliwal, D. S., Lee, K. J., \& Fargher, N. L. (1991). The association between unexpected earnings and abnormal security returns in the presence of financial leverage. Contemporary Accounting Research, 8(1), 20-41.

Dunbar, A., Higgins, D., Phillips, J., \& Plesko, G. (2010). What do measures of tax aggressiveness measure. In Proceedings of the National Tax Association Annual Conference on Taxation (pp. 18-26).

Gallemore, J., Maydew, E., \& Thornock, J. (2012). The reputational costs of tax avoidance and the under-sheltering puzzle. Working Paper, University of North Carolina, University of Washington.

Hanlon, M., \& Heitzman, S. (2010). A review of tax research. Journal of Accounting and Economics, 50(2), 127-178. 
Holthausen, R. W., \& Verrecchia, R. E. (1988). The effect of sequential information releases on the variance of price changes in an intertemporal multi-asset market. Journal of Accounting Research, 26(1), 82-106.

Hoopes, J. L., Mescall, D., \& Pittman, J. A. (2012). Do IRS audits deter corporate tax avoidance? The Accounting Review, 87(5), 1603-1639.

Hope, O. K., Yiqiang. J., \& Tony, K. (2005). Empirical evidence on jurisdictions that adopt IFRS. Journal of International Accounting Research, (5), 1-20.

Kubata, A., Lietz, G. M., \& Watrin, C. (2013). Does Corporate Tax Avoidance Impair Earnings Informativeness?. Working paper, Munster School of Business and Economics. Institute Of Accounting and Taxation.

Lietz, G. M. (2013). Tax avoidance vs. tax aggressiveness: A unifying conceptual framework. Working Paper, University of Münster.

Lisowsky, P., Robinson, L., \& Schmidt, A. (2013). Do publicly disclosed tax reserves tell us about privately disclosed tax shelter activity?. Journal of Accounting Research, 51(3), 583-629.

Martinez, A. L., \& Dalfior, M. D. (2016). Agressividade Fiscal entre Companhias Controladoras e Controladas. Revista da Receita Federal: estudos tributários e aduaneiros, 2(1), 344-362.

Martinez, A. L. (2017). Tax aggressiveness: a literature survey. Revista de Educação e Pesquisa em Contabilidade 11 : 104-21.

Scholes, M. S., Wilson, G. P., \& Wolfson, M. A. (1990). Tax planning, regulatory capital planning, and financial reporting strategy for commercial banks. The Review of Financial Studies, 3(4), 625-650.

Scholes, M., Wolfson, M., Erickson, M., Maydew, E., \& Shevlin, T. (2009). Taxes and Business Strategy: A planning approach, Pearson Prentice-Hall. Upper Saddle River, NJ.

Zimmerman, J. (1993). Taxes and firm size. Journal of Accounting and Economics, 5(1), 119149. 